Ann. Biol. anim. Bioch. Biophys., I974, 14 (3), 437-446.

\title{
INFLUENCE D'HORMONES VASOACTIVES ET DU STRESS SUR LE DÉBIT SANGUIN DANS LA GLANDE MAMMAIRE DE LA CHÈVRE EN LACTATION
}

\author{
A. HOUVENAGHEL et G. PEETERS
}

Laboratoive de Physiologie, Faculté de Médecine vótérinaire, Université de Gand (Belgique)

RÉSUMÉ

Des capteurs de débit électromagnétiques furent implantés au pourtour de l'artère mammaire chez quatorze chèvres en lactation et on procéda à l'étude du débit sanguin de la mamelle et de la pression artérielle. A doses peu élevées, ne dépassant pas $300 \mathrm{mU}$, l'ocytocine entraîne le plus souvent une augmentation du débit sanguin de la mamelle mais la pression artérielle ne subit aucune variation. Des doses plus élevées provoquent une chute du débit accompagnée d'hypertension. La vasopressine entraîne de la vasoconstriction mammaire et une augmentation de la pression artérielle. Sous l'effet de l'histamine et de l'acétylcholine, on enregistre de l'hypotension, une diminution passive et transitoire de la circulation et finalement de la vasodilatation mammaire. La sérotonine diminue le débit sanguin mammaire et augmente la pression artérielle. Le stress émotionnel, déclenché sous l'action de divers types de stimulation, est suivi d'une vasoconstriction au niveau de la mamelle souvent accompagnée d'une élévation de la pression artérielle. Le débit sanguin et la pression artérielle sont stabilisés sous l'effet de la chlorpromazine.

\section{INTRODUCTION}

Le débit sanguin de 1'artère mammaire peut être enregistré dans des conditions physiologiques, à l'aide d'un capteur électro-magnétique, sur animal non anesthésié. Nous avons communiqué récemment les résultats obtenus par administration intraveineuse des catécholamines, des kinines du plasma et de l'angiotensine sur le débit sanguin par cette artère chez des Petits Ruminants en lactation (Houvenagher, I970; Houvenaghel et Peeters, I972). Dans le présent travail nous rapportons les résultats d'une étude faite chez la Chèvre en vue de vérifier l'influence d'autres hormones vasoactives et de facteurs stressants sur le débit sanguin de la mamelle et sur la pression artérielle. 


\title{
MATÉRIEL E'T MÉTHODES
}

\begin{abstract}
Les expériences furent effectuées chez quatorze chèvres, de différentes races, en état de lactation (poids 25 à $50 \mathrm{~kg}$; production de lait : 650 à I $750 \mathrm{ml} / \mathrm{j}$ ). Un capteur électromagnétique est implanté, selon une technique que nous avons récemment décrite (Houvenaghel et Peeters, I972), autour de l'artère d'une moitié du pis (A. pudenda externa). La pression sanguine est enregistrée à l'aide d'un capteur de pression (Statham) au niveau d'une carotide extériorisée. Les hormones vasoactives sont injectées rapidement au moyen d'un tube en polyéthylène, préalablement inséré dans la veine jugulaire. Toutes les substances sont diluées dans du sérum physiologique et injectées dans un volume de I $\mathrm{ml}$ et l'intervalle entre les injections successives est de 5 ou de ro minutes. La plupart des animaux s'habituent rapidement aux conditions expérimentales : il arrive même de les voir ruminer au cours des enregistrements. Les modifications du débit diastolique sont mesurées au moment du pic de l'effet et exprimées en pourcentage par rapport à la valeur contrôle.

Les substances suivantes sont utilisées : l'ocytocine (NobitocineR, Nobilis, Boxmeer); la vasopressine (PitressineR, Parke Davis, London ou l'arginine-vasopressine, AVP, Ferring, Malmö); le chlorhydrate d'acétylcholine (Roche, Bâle) ; la sérotonine, sous forme de sulfate de créatinine (Koch-Light, Colnbrook) ; le chlorhydrate d'histamine (Calif. Corp. Biochem. Res., Los Angeles) et la chlorpromazine (LargactilR, Specia, Paris). Deux types de préparations d'ocytocine sont disponibles : une solution contenant du chlorbutol (Io U d'ocytocine $+2,9 \mathrm{mg}$ de chlorbutol par $\mathrm{ml}$ ) et une autre solution ne renfermant aucune substance préservatrice.
\end{abstract}

\section{RÉSUITATS}

\section{L'ocytocine}

Les résultats varient selon la dose administrée. Au départ nous avons utilisé la préparation d'ocytocine renfermant du chlorbutol. Comme d'après certains auteurs (KATz, I964; Somlyo, Woo et Somlyo, I966) celui-ci possède une faible activité sur le tonus vasculaire, nous avons utilisé ultérieurement la préparation d'ocytocine sans chlorbutol. Dans nos expériences le chlorbutol à des doses de 25 à I $000 \mu g$ ne provoque aucun effet sur le débit mammaire ni sur la pression artérielle.

L'influence de doses peu élevées d'ocytocine (3 à $300 \mathrm{mU}$ ) est étudiée sur huit chèvres. Chez 7 animaux l'ocytocine provoque une augmentation plus ou moins prononcée du débit sanguin au niveau de l'artère mammaire (latence 23 à $43 \mathrm{~s}$ ) sans influencer pour autant la pression artérielle (fig. I). La dose seuil est de ro $\mathrm{mU}$ chez 3 animaux et de $30 \mathrm{mU}$ chez les quatre autres. Cette augmentation du débit persiste de 23 à $155 \mathrm{~s}$. Deux de ces 7 chèvres ont été traitées par une préparation d'ocytocine renfermant du chlorbutol, tandis que les 5 autres ont reçu l'ocytocine seule. Trente effets positifs ont été enregistrés à partir d'ocytocine avec chlorbutol et 80 à partir d'ocytocine sans chlorbutol. Chez une chèvre cependant l'administration de ces doses peu élevées d'ocytocine, ne renfermant pas du chlorbutol, provoque une chute du débit sanguin mammaire (latence 7 à I $8 \mathrm{~s}$; durée Io à $36 \mathrm{~s}$ ).

L'injection de doses plus élevées d'ocytocine sans chlorbutol (I à 24 U) à cinq animaux, nous a permis de constater une chute du débit sanguin au niveau de l'artère mammaire (latence Io à I5 s) en même temps que de l'hypertension (latence 7 à ro s). L'intensité de cette dernière varie de 5 à $30 \mathrm{~mm} \mathrm{Hg}$ (fig. 2). 

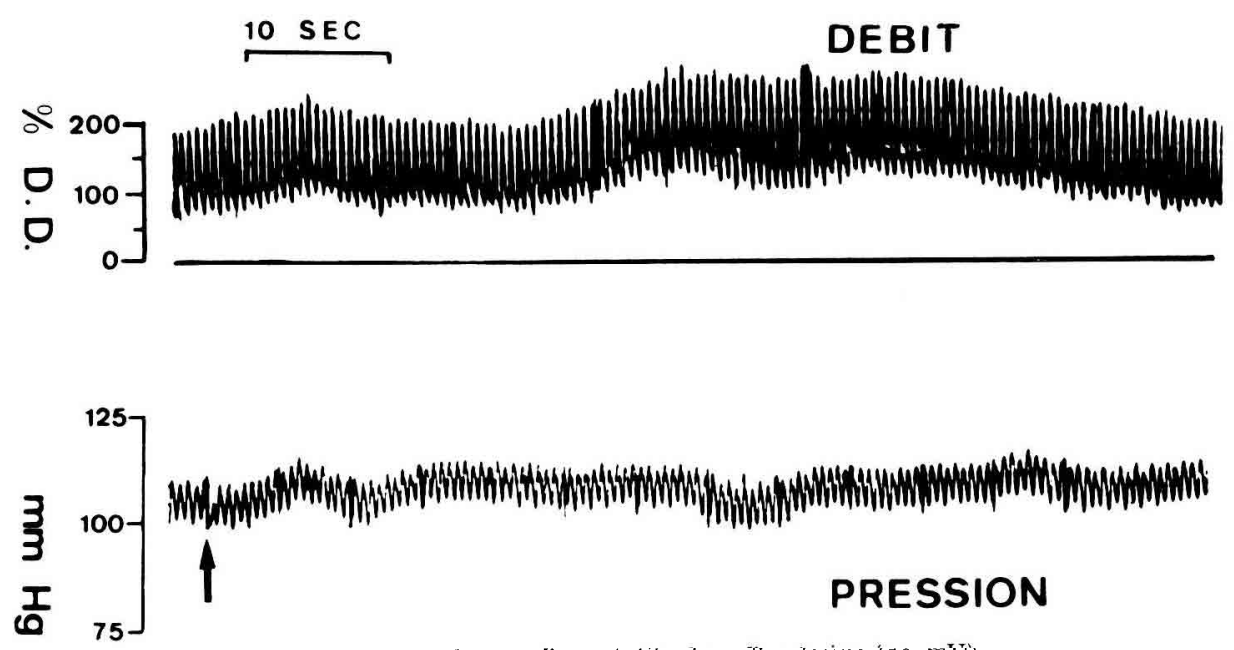

Fig. I. - Influence d'une petite dose d'ocytocine (Io mú) sur le débit sanguin par le pis (courbe supérieure) et sur la pression artérielle (courbe inférieure)

$\uparrow$ : moment de l'injection;

P. roo D.D. : valeur procentuelle du débit diastolique
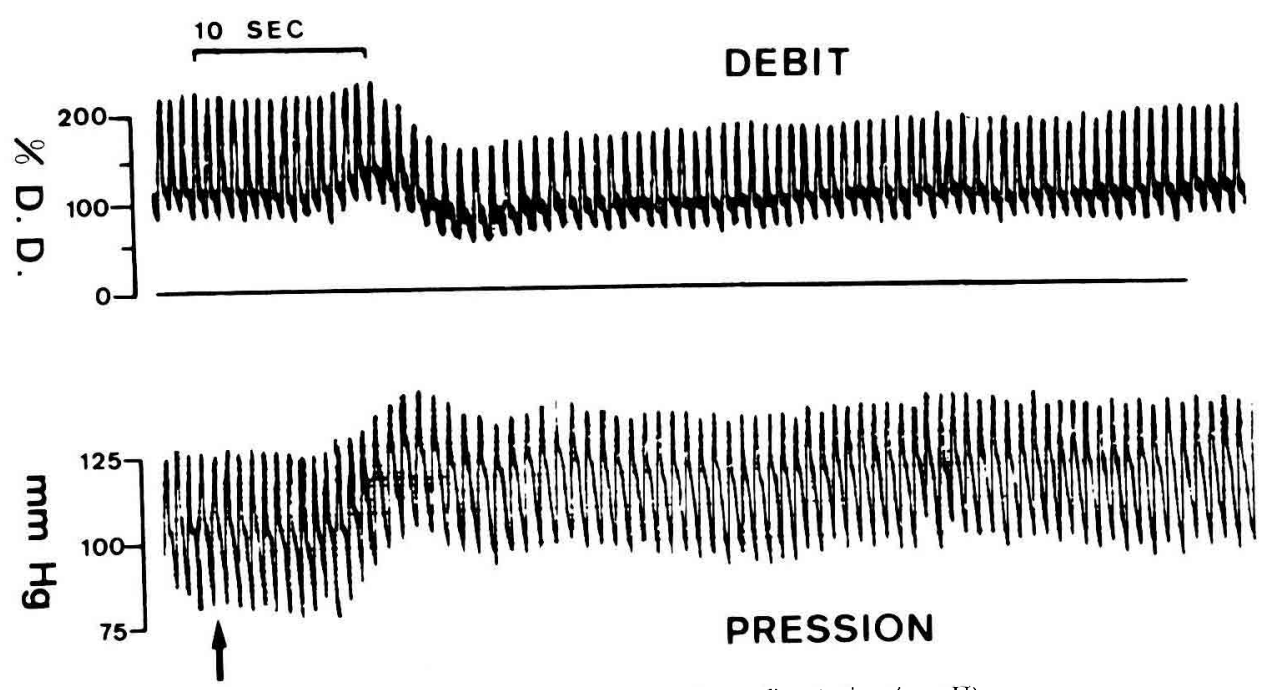

FiG. 2 - Influence d'une dose élevée d'ocytocine $(7,2 \mathrm{U})$ 
La chute du débit mammaire persiste de Io à $\mathrm{r}_{50} \mathrm{~s}$; elle est fonction de la dose administrée. Il existe une corrélation significative $(\log / \log )$ entre d'une part les doses et d'autre part la durée et l'intensité de la vasoconstriction.

\section{2. - La vasopressine}

Les expériences ont porté sur six animaux; chez l'un nous avons employé la Pitressine et chez les cinq autres l'arginine-vasopressine. Les doses administrées ont varié entre Io et I ooo $\mathrm{mU}$. Les deux préparations provoquent une chute du débit sanguin mammaire et une augmentation de la pression artérielle (fig. 3). La dose minima active entraînant une baisse de débit est de $30 \mathrm{mU}$ et celle entraînant une augmentation de pression est de 100 à $300 \mathrm{mU}$. Le débit diminue après un temps de latence de Io à 20 s et cet effet persiste pendant 50 à 300 s selon la dose administrée. Dans plusieurs cas il est difficile de préciser la durée de la diminution du débit sanguin car il ne revient pas complètement à sa valeur initiale. L'intensité de la baisse diastolique du débit varie de Io à $35 \mathrm{p}$. Ioo pour les doses seuils ; une baisse maximale est souvent constatée après injection de I $000 \mathrm{mU}$. Des perfusions intraveineuses d'AVP $(6 \mathrm{mU} / \mathrm{kg} / \mathrm{mn}$ durant $30 \mathrm{mn})$ provoquent une diminution significative du débit sanguin de la mamelle en même temps que de l'hypertension. Ces deux effets persistent pendant toute la durée des perfusions et le retour aux valeurs initiales ne se fait que très lentement à l'arrêt de la perfusion.
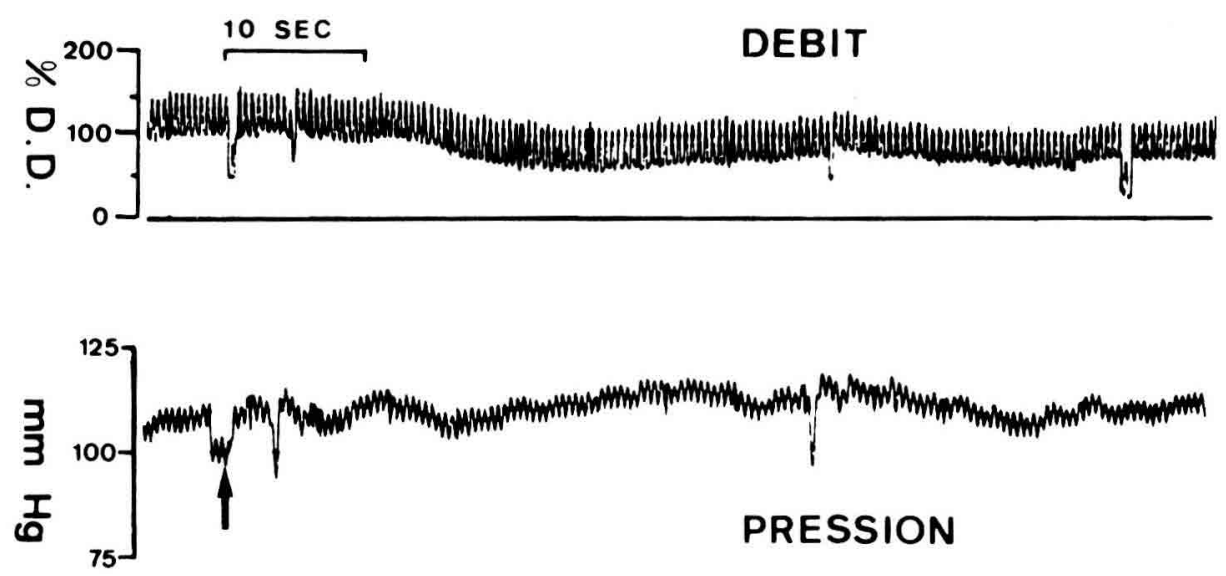

FIG. 3. - Effet de l'arginine-vasopressine (30 $\mathrm{mU})$

\section{3. - L'acétylcholine}

L'acétylcholine, administré aux doses de I,66 à I ooo ng/ $\mathrm{kg}$ chez cinq animaux, entraîne une augmentation du débit sanguin de la mamelle en même temps qu'une chute de la pression artérielle (fig. 4). A la suite de cette baisse de pression, la vasodilatation dans la glande est précédée par une chute passive de courte durée. La dose minima active sur la circulation mammaire varie entre 7,5 et $40 \mathrm{ng} / \mathrm{kg}$. Le plus souvent cette augmentation de débit n'est que de courte durée (Io à $35 \mathrm{~s}$ ).

L'injection intraveineuse de sulfate d'atropine $(0,25 \mathrm{mg} / \mathrm{kg})$ provoque une inhibition transitoire (environ une heure) des effets de l'acétylcholine. 

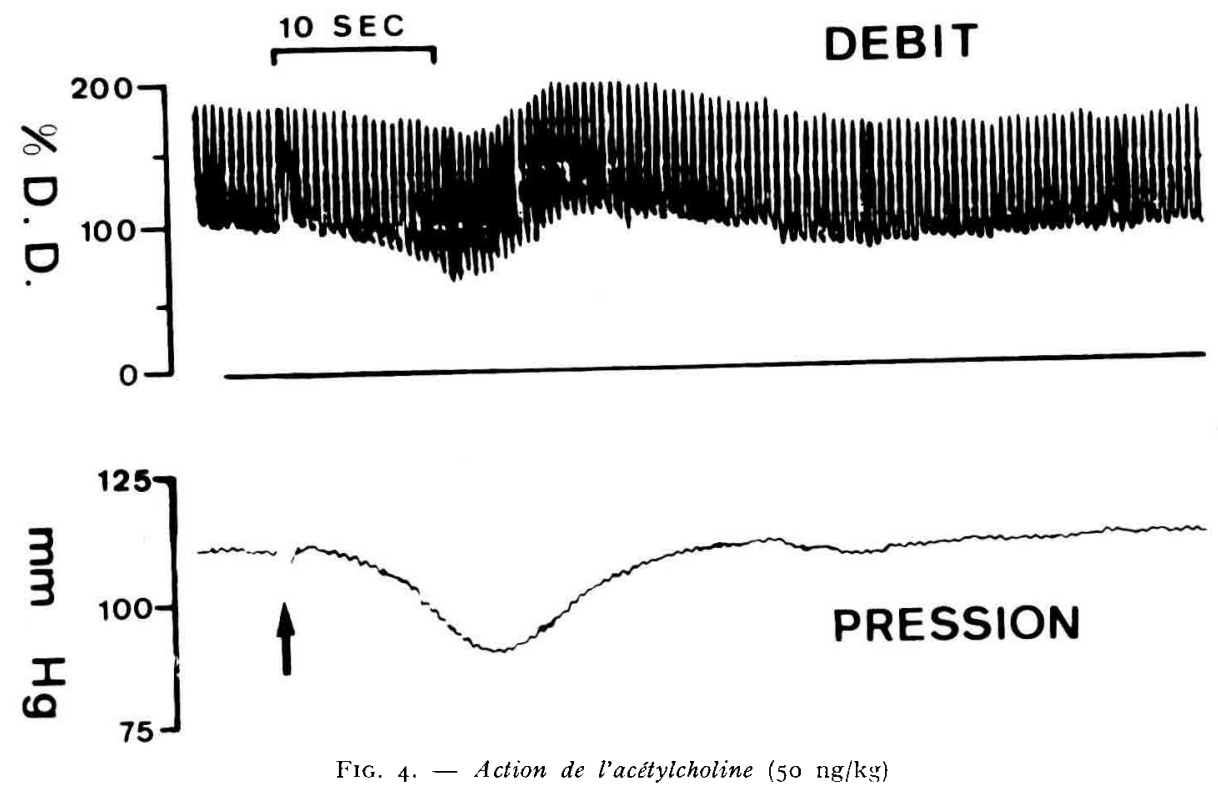

4. - La sérotonine

L'action de la sérotonine fut étudiée chez six animaux; les doses employées ont varié de $0, I_{5}$ et $\mathrm{I} 5 \mu \mathrm{g} / \mathrm{kg}$. Cette substance provoque une chute du débit sanguin au niveau de l'artère mammaire (fig. 5). La dose seuil susceptible d'entraîner une
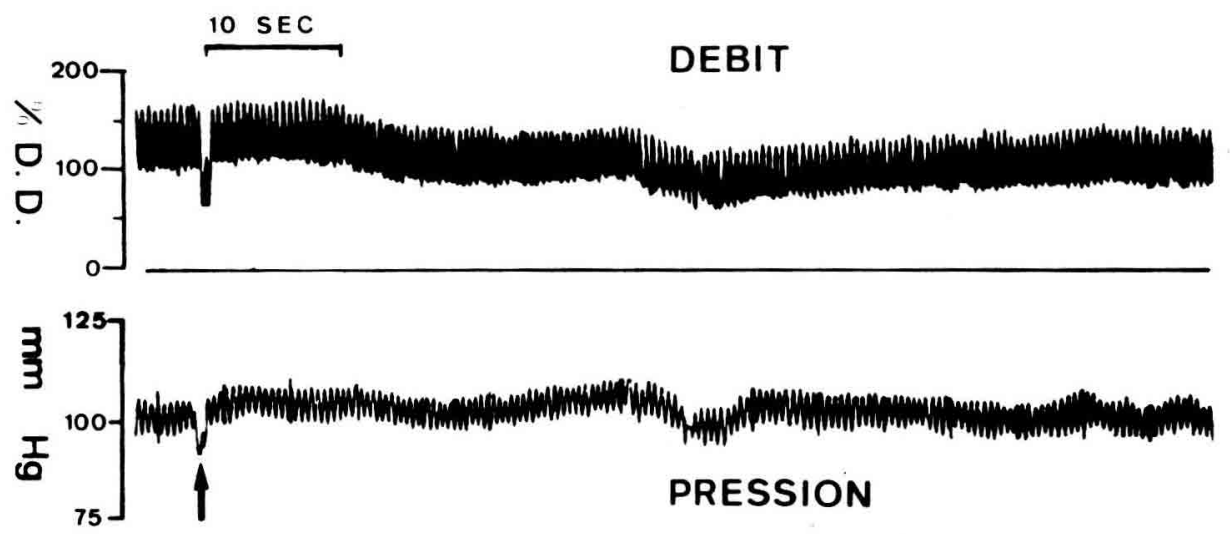

FIG. 5. -- Influence de la sérotonine $(750 \mathrm{ng} / \mathrm{kg})$

baisse du débit diastolique de I3 à $30 \mathrm{p}$. Ioo, s'étend entre 0,35 et $4 \mu \mathrm{g} / \mathrm{kg}$. Chez une chèvre, l'injection de $\mathrm{I} 2 \mu \mathrm{g} / \mathrm{kg}$ a provoqué une baisse maximale du débit diastolique. Si des doses plus élevées sont employées, la vasoconstriction s'accompagne d'hypertension. La durée de l'effet vasoconstricteur varie entre 30 et $450 \mathrm{~s}$. 


\section{5. - L'histamine}

Cette substance fut administrée à cinq chèvres à la dose de 0,0 I5 à Io $\mu \mathrm{g} / \mathrm{kg}$; elle provoque une augmentation du débit sanguin mammaire en même temps qu'une chute de la pression artérielle (fig. 6). La dose minima active varie entre 25 et $120 \mathrm{ng} / \mathrm{kg}$. L'augmentation de débit est généralement précédée d'une chute passive de courte durée consécutive à l'hypotension. La vasodilatation mammaire persiste de Io à $240 \mathrm{~s}$.
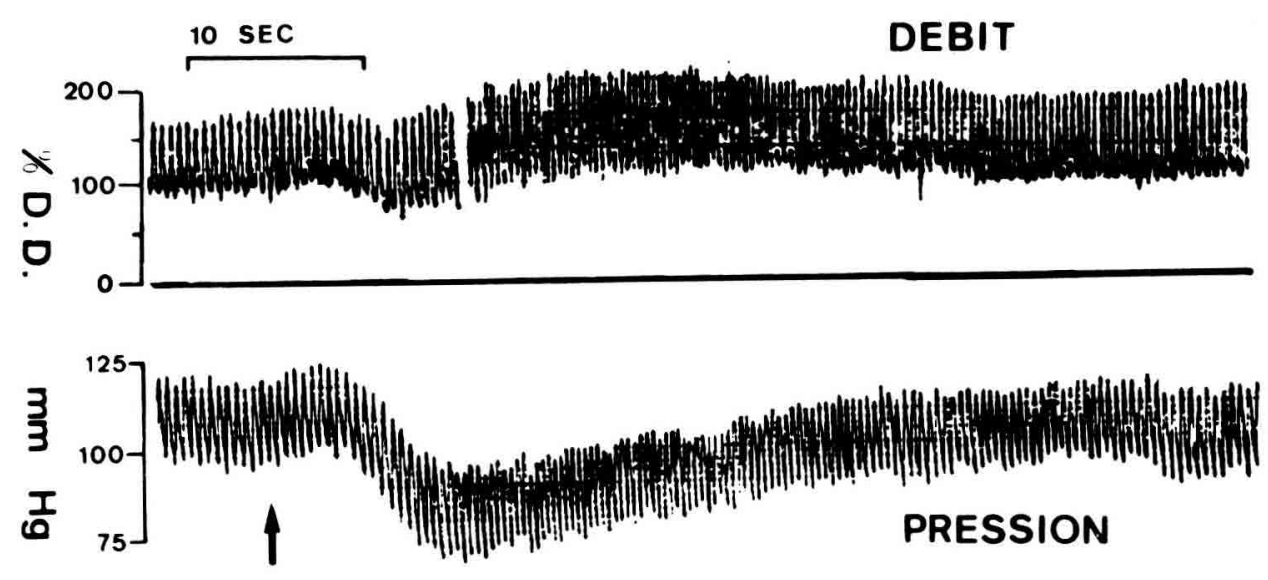

FIG. 6. - Influence de l'histamine ( $\mu \mathrm{g} / \mathrm{kg}$ )

$$
\text { 6. - Le stress }
$$

Nous avons signalé dans un précédent travail, l'action vasoconstrictive élective des catécholamines sur les vaisseaux mammaires de la Chèvre lactante (HouvenaGHEL, I970). Dans ces expériences nous avons souvent constaté que l'état de frayeur des animaux, provoqué par des facteurs divers tels que bruit, entrée de personnes étrangères, etc., entraînait une diminution brusque du débit sanguin mammaire. Dans le présent travail, nous avons étudié l'influence du stress sur le débit sanguin mammaire et sur la pression artérielle. Nous avons eu recours à 3 types de stimulation : battement des mains ; piqûre de la peau du thorax avec une aiguille ; stimulations électriques de faible voltage ( 6 Volts au maximum) dans la région lombaire durant une seconde. Plus de I50 stimulations ont été appliquées chez 6 chèvres. Toutes ces interventions effrayent les animaux et provoquent des réactions de défense. Le plus souvent les chèvres se retirent brusquement et plient les pattes de derrière pendant quelques secondes. Miction et défécation sont fréquemment observées. Les stimulations provoquent une diminution significative du débit sanguin mammaire fréquemment accompagnée d'une augmentation de la pression artérielle. Chez 3 chèvres la baisse du débit présente le plus souvent un aspect biphasique : une chute primaire de courte durée (6 à I5 s), débutant immédiatement après l'application de la stimulation, est suivie d'une baisse secondaire qui est plus prononcée et qui persiste pendant 20 à $200 \mathrm{~s}$ (fig. 7). Pendant la chute primaire la tachycardie est fréquente. Chez les 3 autres animaux la plupart des stimulations ne provoquent 
que la chute primaire. L'intensité de la diminution du débit diastolique varie de 20 à 80 p. Ioo. La pression sanguine commence à augmenter après un temps de latence de o à Io s. L'hypertension, qui atteint une valeur de Io à $30 \mathrm{~mm} \mathrm{Hg}$, persiste pendant Io à $60 \mathrm{~s}$.
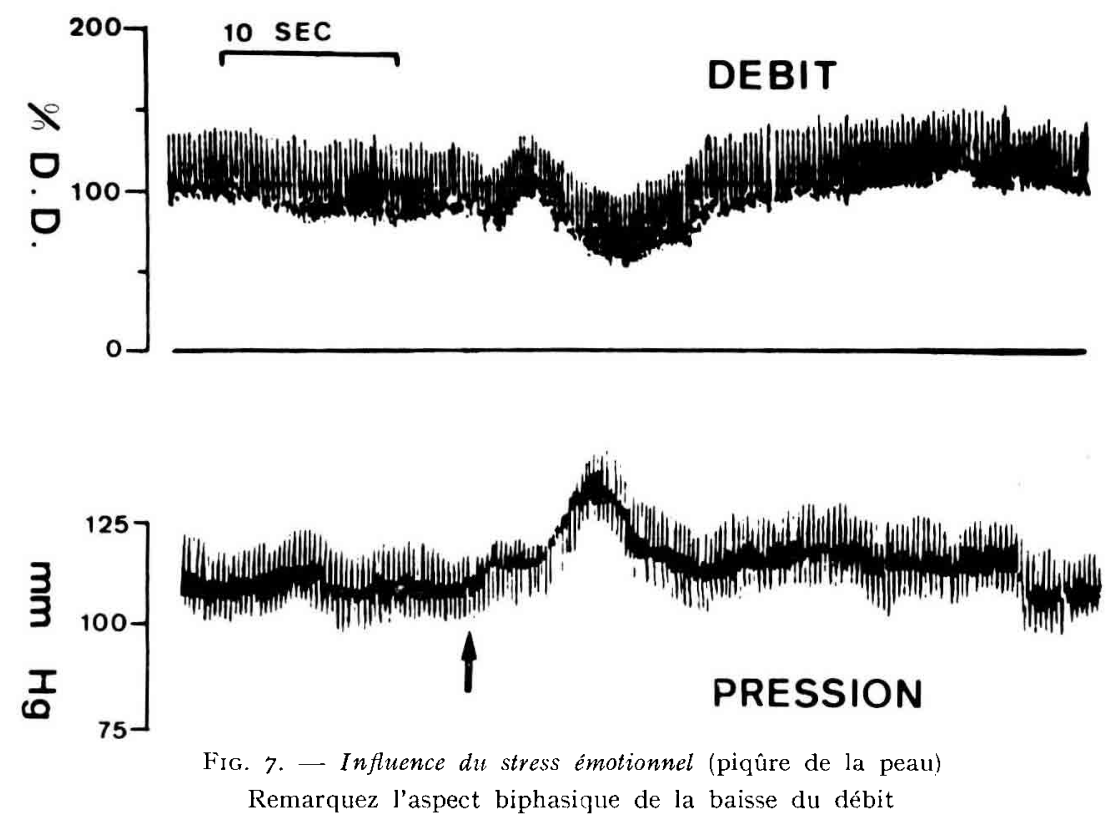

\section{7. - Influence de la chlorpromazine}

Au cours des premiers jours d'enrezistrement, la plupart des chèvres, quoique traitées avec beaucoup de circonspection, se sont montrées inquiètes. Une instabilité du débit sanguin et de la pression artérielle fut constatée. Nous avons alors administré de la chlorpromazine à 7 chèvres et étudié son action sur la circulation mammaire et la pression artérielle après avoir enregistré les deux paramètres pendant ine demi-heure. La chlorpromazine est injectée lentement par voie intraveineuse à la posologie de $0, \mathrm{I}$ à $0,5 \mathrm{mg} / \mathrm{kg}$. Dans $50 \mathrm{p}$. Ioo des expériences le débit sanguin et la pression artérielle se stabilisent de façon significative ; cet effet stabilisant apparaît environ Io mn après l'injection du produit et il persiste souvent pendant plusieurs heures. Pendant toute cette période les animaux restent calmes et réagissent moins aux diverses stimulations qu'avant l'injection. Cette action stabilisante de la chlorpromazine n'a pu être observée chez deux chèvres malgré l'adminıstration de doses atteignant $\mathrm{I} \mathrm{mg} / \mathrm{kg}$.

\section{DISCUSSION}

Des doses faibles d'ocytocine, analogues à celles libérées lors de la traite ou de la tétée, sont capables d'augmenter la circulation sanguine de la mamelle chez la Chèvre. Des effets analogues ont été observés chez la Vache laitière ; la vasodilatation 
dans la glande mammaire au cours de la traite de cette dernière dépend en partie de l'ocytocine (Houvenaghei, Peeters et Verschooten, I973). Nous avons constaté chez quatre de nos chèvres d'expérience, une augmentation du débit sanguin de la mamelle au cours de la traite et le début du phénomène a parfois coïncidé avec le moment de l'éjection du lait. Il apparaît que les vaisseaux sanguins de la Chèvre sont très sensibles à l'action de l'ocytocine (dose seuil Io à $30 \mathrm{mU}$ IV); les cellules myoépithéliales le sont encore davantage, puisque la dose minima active, qui provoque l'éjection du lait, varie entre I et $5 \mathrm{mU}$ (Houvenaghel et PeETERs, I968). De fortes doses pharmacologiques d'ocytocine exercent un effet vasoconstrictif au niveau du pis.

L'AVP provoque une augmentation de la pression sanguine en même temps que de la vasoconstriction de la glande mammaire chez la Chèvre. Si cette hormone entraîne de l'hypertension chez des Vaches, son effet sur la circulation mammaire est cependant variable et dans de nombreux cas, on observe une vasodilatation (DHondr et al., 1973). Dans les deux espèces, bovine et caprine, il est nécessaire de recourir à des doses relativement élevées d'AVP pour obtenir ces effets. Les quantités d'AVP libérées lors du stress, de l'hémorragie, d'un choc ou d'hypotension sont probablement suffisantes pour influencer la circulation mammaire. La traite exerce souvent un effet antidiurétique de type pituitaire chez les Petits Ruminants (PeÉTERs et Debackfre, I963). Si l'AVP est l'élément responsable de cette action, les doses libérées sont cependant trop faibles pour influencer la circulation mammaire. Il est remarquable de constater que l'AVP puisse provoquer, chez les deux espèces, des effets vasoconstricteurs prolongés au niveau du pis.

La pression sanguine diminue sous l'effet de l'acétylcholine chez la Chèvre et chez la Vache et ce phénomène est concomitant de la vasodilatation au niveau de la mamelle (DHondr et al., I973). La dose minimale active, exprimée par $\mathrm{kg}$ de poids, est plus faible chez la Chèvre (7,5-40 ng) que chez la Vache (200-2 ooo ng). Cette différence pourrait s'expliquer éventuellement par le temps de circulation plus court chez la Chèvre et par la teneur en cholinestérase sanguine plus faibles chez la Chèvre que chez la Vache (KoL, I957).

La vasodilatation observée au niveau de la mamelle après l'injection intraveineuse de faibles doses d'histamine concorde avec les observations faites par LINZELL (I950). Cet auteur a constaté une vasodilatation dans la glande mammaire perfusée de Chien et de Chat après injection de $O, I \mu g$ d'histamine dans l'artère.

La sérotonine exerce une action vasoconstrictive au niveau de la mamelle. Il est intéressant d'ailleurs de signaler que des phénomènes de vasoconstriction marquée s'observent au niveau de la glande mammaire isolée de Ruminants perfusée par du sang hépariné. Ce fait peut trouver une explication dans la libération de sérotonine par les plaquettes sanguines lésées au cours de la perfusion. L'administration de substances inhibitrices de la sérotonine telles la dibénamine (PEETERS et MASSAR'T, I952), et l'acide bromolysergique diéthylamide (HARDWICK et LINZELI, I960) augmente de façon spectaculaire le débit sanguin de la glande mammaire isolée et perfusée.

Il est connu depuis de nombreuses années que le stress provoqué par le bruit, la peur ou la douleur, inhibe l'éjection du lait (EI, et PETERSEN, I94I) ; cela résulte sans doute de la stimulation des centres sympatiques de l'hypothalamus. En effet la stimulation électrique de ces centres chez des Lapines en lactation provoque une 
puissante inhibition de l'action de l'ocytocine sur l'éjection du lait. Cet effet inhibiteur est souvent, mais pas toujours, éliminé par la surrénalectomie bilatérale (Cross, I955). Ces résultats laissent présumer que l'action inhibitrice déclenchée par le stress pourrait s'exercer par l'intermédiaire des catécholamines secrétées au niveau des glandes surrénales ou des fibres sympathiques de la glande mammaire. Nos expériences montrent que la circulation sanguine de la mamelle chez la Chèvre peut être inhibée par le stress. La baisse primaire du débit mammaire qui débute immédiatement après l'application de la stimulation, peut être considérée en grande partie comme un effet mécanique, provoqué par le changement de position de l'animal. D'autre part la tachycardie et l'hypertension, souvent observées durant la baisse primaire, plaident en faveur d'une activation du système nerveux sympatique, à la suite de la stimulation. Il nous semble probable, que la baisse secondaire observée après une période de latence plus longue, est provoquée par un mécanisme hormonal, c'est-à-dire par les catécholamines libérées par les glandes surrénales ou l'arginine vasopressine libérée par la neurohypophyse. Il est clair qu'une étude plus approfondie du mécanisme du stress sur la circulation mammaire est nécessaire.

$\mathrm{Au}$ cours des premiers jours d'enregistrement, la chlorpromazine exerce dans de nombreux cas, une action stabilisante sur la circulation sanguine mammaire. Cet effet s'explique par les propriétés sédatives, sympathicolytiques et adrénolytiques de cette substance.

Reçu pour publication en janvier 1974.

\title{
REMERCIEMENTS
}

Ces recherches ont été subventionnées par l'Institut pour l'Encouragement de la Recherche Scientifique dans l'Industrie et l'Agriculture (I. R. S. I. A.) et par le Fonds National de la Recherche Scientifique (F. N. R. S.).

Nous remercions $M^{m e}$ G. Vrancken, Mme J. Smekens et M. G. Rasschaert pour leur collaboration technique ainsi que MM. A. Coppieters, G. Deboevere et E. Van der Elstraeten, qui ont soigné les animaux d'expérience. Nous sommes très reconnaissants envers les laboratoires Nobilis, Boxmeer, qui ont mis gracieusement l'ocytocine, ne renfermant pas du chlorbutol, à notre disposition ainsi qu'envers les laboratoires Ferring, Malmö, qui nous ont procuré l'AVP.

\section{SUMMARY}

\author{
INFLUENCE OF VASOACTIVE HORMONES \\ AND OF STRESS ON BLOOD FLOW THROUGH THE UDDER \\ OF THE LACTATING GOAT
}

In 14 lactating goats bearing electromagnetic flowprobes implanted around the artery of one mammary gland, the influence of IV injections of vasoactive hormones and of stress on blood flow through the udder and arterial pressure was studied. Small doses of oxytocin (up to $300 \mathrm{mU}$ ) mostly induced an increase of blood flow through the udder without any change in arterial pressure; larger doses of oxytocin however provoked a decrease of blood flow accompanied by hypertension. Vasopressin caused vasoconstriction in the udder and an increase of arterial pressure. Injection of histamine and acetylcholine resulted in an increase of blood flow preceded by a short lasting passive decrease owing to their hypotensives effects. Serotonin induced 
vasoconstriction in the udder and an increase of arterial pressure. Application of stimuli causing stress provoked inhibition of blood flow through the gland often accompanied by an increase of blood pressure. Both mammary blood flow and arterial pressure could be stabilized by IV injection of chlorpromazine.

\section{RÉFÉRENCES BIBLIOGRAPHIQUES}

Cross B. A., 1955. The hypothalamus and the mechanism of sympathetico-adrenal inhibition of milk ejection. J. Endocr., 12, 15-28.

Dhondt G., Houvenaghel A., Peeters G., Verschooten F., I973. Influence of vasoactive hormones on blood flow through the mammary artery in lactating cows. Arch. int. Pharmacodyn., 204, 89-104.

Ely F., Petersen W. E., I94I. Factors involved in the ejection of milk. J. Dairy Sci., 24, 2 I 1-233.

HaRdwick D. C., Linzell J. L., I960. Some factors affecting milk secretion by the isolated perfused mammary gland. J. Physiol. Lond., 154, 547-57r.

Houvenaghel A, r97o. Action of catecholamines on blood flow through the mammary gland in unanesthetized lactating small ruminants. Arch. int. Pharmacodyn., 186, rgo-rgr.

Houvenaghel A., Peeters G., ig68. Influence de la bradykinine sur l'éjection du lait chez la Brebis et la Chèvre. Arch. int. Physiol. Biochim., 76, 647-657.

Houvenaghel A., Peeters G., 1972. Action of angiotensin and plasmakinins on blood flow through the mammary artery in lactating small ruminants. Arch. int. Pharmacodyn., 200, 320-329.

Houvenaghel A., Peeters G., Verschooten F., 1973. Influences of manual udder stimulation and oxytocin on mammary artery blood flow in lactating cows. Arch. int. Pharmacodyn. 205, I24-I33.

Katz R. L., 1964. Antiarrythmic and cardiovascular effects of synthetic oxytocin. Anesthesiology, 25, 653-66r.

KolB E., I957. Untersuchungen über das Vorkommen von Cholinesterase im Blut und Serum von Haustieren (Rind, Schaf, Ziege, Pferd, Schwein, Hund) und ihre Beeinflussung durch Kontaktinsektizide (Metasystox und Diazinon). Z Zbl. VetMed., 4, 967-982.

LinzelL J. L., r950. Vasomotor nerve fibres to the mammary glands of cat and dog. Quart. J. Exp. Physiol., 35, 295-319.

Peeters G., Debackere M, I963. Influence de la distension du vagin et de la traite ou de la succion sur la diurése chez la Brebis et la Chèvre. J. Physiol. Paris, 55, 48r-493.

Peeters G., Massart L., 1952. Fat synthesis in the perfused lactating cow's udder. Arch. int. Pharmacodyn., 91, 388-398.

Somlyo A. V., Woo C. Y., Somlyo A. P., I966. Effect of magnesium on posterior pituitary hormone action on vascular smooth muscle. Am. J. Physiol., 210, 705-7r4. 